\title{
J. PELlaUmail
}

\section{Modélisation avec pivot pour une loi générale}

Revue française d'automatique, d'informatique et de recherche opérationnelle. Recherche opérationnelle, tome 19, n 3 (1985), p. 221-231.

<http://www.numdam.org/item?id=RO_1985_19_3_221_0>

(C) AFCET, 1985, tous droits réservés.

L'accès aux archives de la revue « Revue française d'automatique, d'informatique et de recherche opérationnelle. Recherche opérationnelle » implique l'accord avec les conditions générales d'utilisation (http://www.numdam.org/ legal.php). Toute utilisation commerciale ou impression systématique est constitutive d'une infraction pénale. Toute copie ou impression de ce fichier doit contenir la présente mention de copyright.

\section{Numdam}

Article numérisé dans le cadre du programme

Numérisation de documents anciens mathématiques

http://www.numdam.org/ 


\title{
MODÉLISATION AVEC PIVOT POUR UNE LOI GÉNÉRALE (*)
}

\author{
par J. Pellaumail ( ${ }^{1}$ )
}

\begin{abstract}
Résumé. - On établit un algorithme qui permet de calculer directement les probabilités stationnaires d'un réseau comprenant deux stations, l'une des stations étant de type PH.

Mots clés : Files d'attente; Modélisation-markovienne; distribution stationnaire.

Abstract. - An algorithm is stated that allows to compute the steady-states probabilities for a queuing network with two stations.
\end{abstract}

Keywords: Queue; Cox model: steady-state probabilities.

\section{INTRODUCTION}

L'objet essentiel de cette étude est de donner un algorithme permettant de calculer directement - sans inversion de matrice, ni calcul de valeurs propres... - les probabilités stationnaires d'un réseau de files d'attente constitué de deux stations $S_{1}$ et $S_{2}$.

La station $S_{1}$ a un seul serveur de loi de service $\mathrm{PH}$ - c'est-à-dire que le processus de service est un processus de renouvellement et que la durée du service est modélisable par un sous-réseau markovien. La " vitesse " de service du serveur peut dépendre non seulement de l'état fictif mais aussi du nombre de clients dans la station $S_{1}$. Dans la station $S_{2}$ la loi de service est exponentielle mais le taux de service peut dépendre du nombre de clients dans la station (cas de plusieurs serveurs par exemple). En fait, ce taux de service en $S_{2}$ peut aussi dépendre de l'état fictif en $S_{1}$.

Dans la suite $(i, j, n)$ désignera un état pour lequel il y a $(n-1)$ clients en $S_{1}$, le couple $(i, j)$ correspondant à l'état fictif du serveur de $S_{1}$.

(*) Reçu juin 1984 .

(1) I.N.S.A., 20, avenue des Buttes-de-Coësmes, 35043 Rennes Cedex.

R.A.I.R.O. Recherche opérationnelle/Operations Research, 0399-0559/85/03 221 11/\$3.10 (C) AFCET-Gauthier-Villars. 
L'originalité essentielle de l'étude réside dans la modélisation de la loi de service de $S_{1}$, un état fictif privilégié jouant un rôle de "pivot " et dans l'algorithme utilisant cette modélisation. La taille mémoire minimum pour mettre en œuvre cet algorithme est de l'ordre de deux fois le nombre d'états fictifs.

Le plan adopté est le suivant :
A. Description du modèle;
B. Exemple;
C. Liaison avec la modélisation de Cox;
D. Probabilités stationnaires;
E. Autre relation de récurrence;
F. Fonction génératrice;

Conclusion.

\section{A. DESCRIPTION DU MODÈLE}

La liaison entre le modèle "abstrait " donné dans ce paragraphe et le système concret évoqué dans l'introduction sera précisée au paragraphe B qui suit.

Soit $I$ un entier avec $I \geqq 1$ : en fait le cas $I=1$ correspond à une loi exponentielle et a peu d'intérêt par la suite. Pour tout entier $i$ avec $2 \leqq i \leqq I$ soit $m(i)$ un entier, $m(i) \geqq 2$. On pose $m(1):=1$. Soit $E^{\prime}$ la partie de $(\mathbb{N} \times \mathbb{N} \times \mathbb{N})$ définie par :

$(i, j, n)$ appartient à $E^{\prime}$ si et seulement si $n \geqq 1,1 \leqq i \leqq I$, et $1 \leqq j \leqq m(i)$. Ou bien on considère un réseau ouvert et l'ensemble $E$ des états est l'ensemble $E^{\prime}$ défini ci-dessus. Ou bien le nombre total $(B-1)$ de clients est fini et l'ensemble $E$ des états est l'ensemble des éléments $(i, j, n)$ de $E^{\prime}$ tels que $n \leqq B$.

Le modèle considéré est un processus markovien $\left(X_{t}\right)_{t \in T}$ qui admet $E$ comme ensemble des états et qui évolue en temps continu : en fait, on ne s'intéressera qu'à la loi stationnaire de ce processus qui sera supposé homogène et ergodique. La loi d'évolution est caractérisée par le générateur infinitésimal, autrement dit, elle est complètement déterminée par la fonction positive $g$ définie - pour les éléments $\left(e, e^{\prime}\right)$ de $(E \times E)$ tels que $e \neq e^{\prime}$ par :

$$
g\left(e, e^{\prime}\right)=\lim _{h \downarrow 0} \cdot \frac{1}{h} \operatorname{Prob}\left[X_{t+h}=e^{\prime} \mid X_{t}=e\right] .
$$

Nous allons maintenant définir cette fonction $g$.

R.A.I.R.O. Recherche opérationnelle/Operations Research 
Soit $s$ et $d$ deux fonctions positives définies sur $E$. De plus, pour tout couple d'entiers $(i, n)$ avec $1 \leqq i \leqq I$ et $n \geqq 1$ (resp. $1 \leqq n \leqq B$ ) si le réseau est ouvert (resp. fermé), soit un réel positif $a(i, n)$. On pose alors :

(1) quel que soit $(i, j, n)$ élément de $E$ :

$$
g[(i, j, n),(i, j, n+1)]=s(i, j, n),
$$

si le réseau est fermé, on doit avoir $s(i, j, B)=0$;

(2) quel que soit $(i, n)$ avec $2 \leqq i \leqq I$ et $n \geqq 2$ :

$$
g[(1,1, n),(i, m(i), n)]=a(i, n) ;
$$

(3) quel que soit $(i, j, n)$ avec $2 \leqq i \leqq I, 2 \leqq j \leqq m(i), n \geqq 2$ :

$$
g[(i, j, n),(i, j-1, n)]=d(i, j, n) ;
$$

(4) quel que soit $(i, n)$ avec $2 \leqq i \leqq I$ et $n \geqq 2$ :

$$
g[(i, 1, n),(1,1, n-1)]=d(i, 1, n) ;
$$

(5) quel que soit $n \geqq 2$ :

$$
g[(1,1, n),(1,1, n-1)]:=a(1, n) ;
$$

(6) $g\left(e, e^{\prime}\right)=0$ dans tous les autres cas.

On pose $d(1,1, n):=\sum_{i=1}^{1} a(i, n)$.

\section{B. EXEMPLE}

Nous allons maintenant donner un exemple "physique " qui correspond à un cas particulier du modèle " abstrait " précédent. Plus précisément, reprenons le modèle précédent en supposant que $s$ ne dépend que de $n$ et que $a$ et $d$ ne dépendent que du couple $(i, j)$.

L'exemple "physique " est celui d'un réseau fermé comprenant deux stations $S_{1}$ et $S_{2}$. En $S_{1}$ il y a un seul serveur, la discipline de service est PAPS et la loi de service est de type PH ( $c f$. introduction). Un état est caractérisé par un triplet $(i, j, n)$ où $(i, j)$ correspond à l'état fictif du serveur de $S_{1}$ et $n$ est le nombre de clients en $S_{1}$ plus un. En $S_{2}$ il y a $k=B-n$ clients (où $B$ est une constante) et la loi de service est exponentielle : le taux de service $\mu(k)=\mu(\mathrm{B}-n)=s(n)$ peut dépendre du nombre de clients dans $\mathrm{S}_{2}$. 
Notons que toutes les notations ont été choisies pour faciliter la programmation en Fortran IV standard (pas d'indice nul ou négatif, indices croissants dans les boucles, etc.) : c'est pourquoi le nombre de clients en $S_{1}$ est appelé $(n-1)$ et non pas $n$.

L'originalité de cette étude réside dans la modélisation de la loi de service en $S_{1}$ qui se fait par l'intermédiaire des couples $(i, j):$ donnons l'interprétation " physique » de l'évolution de l'état fictif.

Le couple $(i, j)$ définit l'état " fictif » du serveur; si $n=1$ (station vide), cet état fictif est $(1,1)$ et il reste $(1,1)$ tant qu'il n'y a pas de nouveau client. Quand la station n'est pas vide $(n \geqq 2)$, le serveur est actif et son état fictif suit son évolution propre : pour $2 \leqq i \leqq I$, il passe de l'état $(1,1)$ à l'état $(i, m(i))$ avec le " taux " $a(i)$; une fois qu'il est sur la " chaîne " associée à $i$, il passe de l'état $(i, m(i))$ à l'état $(\mathrm{i}, \mathrm{m}(i)-1)$ avec le taux $d(i, m(i))$, et ainsi de suite : de l'état $(i, k)$ à l'état $(i, k-1)$ avec le taux $d(i, k)$, etc et ceci jusqu'à l'état $(i, 1)$. Une fois arrivé à l'état $(i, 1)$, le serveur revient à l'état fictif $(1,1)$ avec le taux $d(i, 1)$.

Ensuite, l'évolution reprend comme indiqué plus haut (sauf si la station est vide). De plus, il peut y avoir un retour immédiat (feedback) en l'état $(1,1)$ avec le taux $a(1)$.

Enfin, on considère qu'un client est servi à chaque fois que le serveur repasse par l'état fictif $(1,1)$, soit par feedback, soit en venant d'un état $(i, 1)$. On note que immédiatement après la fin du service d'un client, l'état fictif est nécessairement $(1,1)$. C'est cette propriété qui conduit à des simplifications appréciables dans les calculs. L'état fictif $(1,1)$ joue un rôle de " pivot ». Dans la suite, cette modélisation sera appelée $P$-modélisation (modélisation avec « pivot »).

\section{LIAISON AVEC LA MODELISATION DE COX}

Dans [Cox], Cox a proposé une modélisation par états fictifs dont il est facile de vérifier qu'elle permet d'approcher, autant qu'on le veut, n'importe quelle loi de service. Soit $I$ le nombre d'états fictifs de cette modélisation de Cox, le taux de service étant $\lambda(i)$ dans l'état fictif $i$ et la probabilité de finir le service juste après l'état fictif $i$ étant $1-\alpha(i)$. La transformée de Laplace de la loi de service vaut $(\alpha(I):=0)$ :

$$
\frac{\lambda(1)}{s+\lambda(1)}\left\{1-\alpha(1)+\alpha(1) \frac{\lambda(2)}{s+\lambda(2)}\{1-\alpha(2) \ldots\} \frac{\lambda(I)}{s+\lambda(I)}\right\} .
$$

R.A.I.R.O. Recherche opérationnelle/Operations Research 
Une façon (qui n'est pas nécessairement la meilleure!) de construire une $P$-modélisation associée est alors la suivante : on pose $a(1):=[1-\alpha(1)] \lambda(1)$ et, pour $2 \leqq i \leqq I, m(i):=i-1$ et

$$
a(i):=[1-\alpha(i)] \lambda(i) \prod_{k=1}^{i-1} \alpha(k) \quad \text { et } \quad d(i, j):=\lambda(j) .
$$

On vérifie immédiatement que ceci modélise la même loi de service que le réseau de Cox donné précédemment : la $P$-modélisation permet donc, comme la modélisation de Cox, d'approcher, autant qu'on le veut, n'importe quelle loi donnée.

En fait, la $P$-modélisation est beaucoup plus souple que la modélisation de Cox : prenons comme exemple le cas où la transformée de Laplace vaut :

$$
\alpha \frac{\lambda}{s+\lambda}\left\{1+\beta\left(\frac{\mu}{s+\mu}\right)^{k}\right\}
$$

avec $k$ assez grand $(k \simeq 10)$. Cet exemple est un bon exemple " test " car très " contrasté " puisque c'est un mélange d'une loi exponentielle (aléatoire "pur ») et d'une loi à durée de service presque constante (Erlang- $k$ convolée avec la loi exponentielle précédente).

La $P$-modélisation est immédiate " exacte " et comporte $(k+1)$ états fictifs; la modélisation par un réseau de Cox ne peut être qu'approchée et une telle approximation ne sera " valable que s'il y a au moins $(k+1)^{2}$ états fictifs. Indiquons aussi qu'il y a deux cas où la P-modélisation a une forme particulièrement simple. Dans ces deux cas, les calculs ultérieurs se simplifient notablement.

$1^{\text {er }}$ cas : Erlang généralisé.

C'est le cas où la transformée de Laplace est de la forme :

$$
\alpha \frac{\lambda}{s+\lambda}\left\{1+\beta \prod_{k=1}^{m} \frac{\mu(k)}{s+\mu(k)}\right\} .
$$

Dans ce cas, on peut prendre $I:=2$, c'est-à-dire qu'il n'y a qu'une seule "chaîne ": $a(1):=\alpha \lambda ; \quad a(2):=\beta \lambda ; \quad m(2):=m ; \quad$ pour $1 \leqq k \leqq m$,

$$
d(2, k):=\mu(k) \text {. }
$$

$2^{2}$ cas : Hyperexponentiel généralisé.

C'est le cas où la transformée de Laplace de la loi de service peut s'écrire :

$$
\mathscr{L}(s)=\beta_{1} \frac{\mu(2)}{s+\mu(2)}+\beta_{2}\left[\frac{\mu(2)}{s+\mu(2)}\right]^{2}+\sum_{k=3}^{1} \beta_{k} \frac{\mu(k)}{s+\mu(k)},
$$

vol. $19, \mathrm{n}^{\circ} 3$, août 1985 
où $\left(\mu(k)=\mu_{k}\right), 2 \leqq k \leqq I$ et $\left(\beta(k)=\beta_{k}\right), 1 \leqq k \leqq I$ sont deux familles de réels satisfaisant aux conditions suivantes :

$\beta_{1} \geqq 0, \quad \beta_{2} \geqq 0, \quad \sum_{i=1}^{I} \beta_{i}=1, \mu_{2}>0 \quad$ et, quel que soit $k \geqq 3, \quad \mu_{k}>0$ et $\beta_{k}\left(\mu_{2}-\mu_{k}\right)>0$.

Dans ce cas on pose :

$$
\mu_{1}:=\mu_{2} ; \quad \alpha_{2}:=\beta_{2} ; \quad \alpha_{1}:=\beta_{1}+\sum_{k=3}^{I} \beta_{k} \mu_{k} / \mu_{2}
$$

et pour $k \geqq 3, \alpha_{k}:=\beta_{k}\left(\mu_{2}-\mu_{k}\right) / \mu_{2}$; pour $k \geqq 1, a(k):=\alpha_{k} \mu_{1}$ et surtout, pour $k \geqq 2, m(k):=1$; enfin, pour $k \geqq 2, d(k, 1):=\mu_{k}$.

Il y a un cas où les équations ultérieures se simplifient encore plus, c'est le cas où $I=2$ et $m(2)=1$; dans ce cas, la transformée de Laplace de la loi de service est de la forme $\{\lambda /(s+\lambda)\}\{(\alpha+\beta)(\mu /(s+\mu))\}$; on pose $a(1):=\alpha \lambda$, $a(2):=\beta \lambda$ et $d(2,1):=\mu$.

\section{PROBABILITÉS STATIONNAIRES}

Rappelons qu'on a supposé que le processus considéré est ergodique. Pour tout élément $(i, j, n)$ de $E$, appelons $p^{\prime}(i, j, n)$ la probabilité stationnaire d'être dans l'état $(i, j, n)$. On suppose $p^{\prime}(1,1,1) \neq 0$ et, pour tout élément $(i, j, n)$ de $E,(\mathrm{~d}+\mathrm{s})(i, j, n)>0$. Posons :

$$
p(i, j, n):=p^{\prime}(i, j, n) / p^{\prime}(1,1,1)
$$

Pour $n \geqq 2$, on a les équations suivantes :

Pour $i \neq 1$ et $1 \leqq \mathrm{k}<\mathrm{m}(\mathrm{i})$ :

$$
[p(d+s)](i, k, n)=(p d)(i, k+1, n)+(p s)(i, k, n-1) .
$$

Pour $i \neq 1($ et $k=m(i))$ :

$$
[p(d+s)](i, m(i), n)=p(1,1, n) a(i, n)+(p s)(i, m(i), n-1) .
$$

Pour $i=1$, ce qui implique $k=1$ :

$$
\begin{aligned}
{[p(d+s)](1,1, n-1)=p(1,1, n) a(1, n)+(p s)(1,1, n-2) } & \\
& +\sum_{i=2}^{I}(p d)(i, 1, n) .
\end{aligned}
$$

R.A.I.R.O. Recherche opérationnelle/Operations Research 
On pose $q(n):=p(1,1, n)$; pour $i \neq 1$ et $0 \leqq k<m(i)$, on a :

$$
\begin{aligned}
p(i, m(i)-k, n)=q & (n) \frac{a(i, n)}{d(i, m(i)-k, n)} \prod_{h=m(i)-k}^{m(i)}\left(\frac{d}{d+s}\right)(i, h, n) \\
& +\sum_{j=m(i)-k}^{m(i)} \frac{(p s)(i, j, n-1)}{d(i, m(i)-k, n)} \prod_{h=m(i)-k}^{j}\left(\frac{d}{d+s}\right)(i, h, n) .
\end{aligned}
$$

En effet, la relation (2) montre que la relation (4) est satisfaite pour $k=0$, on prouve alors la relation (4) par récurrence croissante sur $k$ en utilisant la relation (1).

Pour $k=m(i)-1$, l'équation (4) donne :

$$
\begin{aligned}
p(i, 1, n)=q(n) \frac{a(i, n)}{d(i, 1, n)} \sum_{j=1}^{m(i)}( & \left.\frac{d}{d+s}\right)(i, j, n) \\
& \quad+\sum_{j=1}^{m(i)} \frac{(p s)(i, j, n-1)}{d(i, 1, n)} \prod_{h=1}^{j}\left(\frac{d}{d+s}\right)(i, h, n) .
\end{aligned}
$$

On pose :

$$
a^{\prime}(n):=a(1, n)+\sum_{i=2}^{I} a(i, n) \prod_{j=1}^{m(i)}\left(\frac{d}{d+s}\right)(i, j, n) .
$$

La relation (5) reportée dans l'équation (3) donne alors :

$$
\begin{aligned}
a^{\prime}(n) q(n)=q(n-1)(d+s) & (1,1, n-1)-q(n-2) s(1,1, n-2) \\
& -\sum_{i=2}^{I} \sum_{j=1}^{m(i)}(p s)(i, j, n-1) \prod_{h=1}^{j}\left(\frac{d}{d+s}\right)(i, h, n)
\end{aligned}
$$

Par ailleurs, $q(0)=0$ et $\mathrm{q}(1)=1$ et, pour $i \neq 1, p(i, j, 1)=0$; autrement dit, pour $n=2$, on connait $q(n-2), q(n-1)$ et $p(i, j, n-1)$. Raisonnons par récurrence croissante sur $n$ et supposons que l'on connaisse $q(n-2), q(n-1)$ et $p(i, j, n-1)$; la relation (7) permet de calculer $q(n)$; la relation (2) permet de calculer $p(i, m(i), n)$; la relation (1) permet de calculer $p(i, m(i)-k, n)$ par récurrence croissante sur $k$. On a donc su calculer $q(n-1), q(n)$ et $p(i, j, n)$ ce qui montre qu'on a bien un algorithme qui permet de calculer toutes les probabilités stationnaires (sans inversion de matrice, sans calcul de valeurs propres, etc.).

Si on ne tient pas compte de la taille mémoire nécessaire pour les paramètres $a, s$ et $d$, (ces paramètres étant en général, donné sous forme de formules 
explicites) et si on n'a pas besoin de garder en mémoire toutes les probabilités stationnaires (cas où seul nous intéresse $\sum_{i, j} p^{\prime}(i, j, n)$ par exemple), on note que l'algorithme proposé nécessite une taille mémoire de l'ordre de $\sum_{i=2}^{I} 2[1+m(i)]$ (ce qui est très peu); en effet, le calcul de $p(i, j, n+1)$ ne nécessite que la connaissance de la famille $(p(i, j, n))_{i, j}$ et celle de $q(n-1)$.

\section{E. AUTRE RELATION DE RECURRENCE}

L'algorithme proposé précédemment donnant lieu à peu de calculs est très précis : néanmoins, il est bien connu que les algorithmes les plus surs sont ceux qui ne font pas intervenir de différence $\gamma=\alpha-\beta$ : en effet, si $\gamma$ est très petit par rapport à $\alpha$ et $\beta$, les erreurs dues aux calculs peuvent devenir considérables. Il est donc intéressant de noter que :

$$
q(n)=\left\{(p s)(1,1, n-1)+\sum_{i=2}^{I} \sum_{j=1}^{m(i)}(p s)(i, j, n-1) w(i, j, n)\right\} / a^{\prime}(n),
$$

où :

$$
w(i, j, n):=1-\prod_{h=1}^{j}\left(\frac{d}{d+s}\right)(i, h, n) \geqq 0 .
$$

Preuve : Rappelons que $a^{\prime}(n)$ est défini en (6). On a la relation suivante : (cf. [Mar] ou [Ke1]) :

$$
\begin{aligned}
&(p s)(1,1, n-1)+\sum_{1=2}^{I} \sum_{j=1}^{m(i)}(p s)(i, j, n-1) \\
&=p(1,1, n) a(1, n)+\sum_{i=2}^{I}(p d)(i, 1, n) .
\end{aligned}
$$

Les relations (5) reportées dans (9) donnent alors la relation (8).

\section{F. FONCTION GENERATRICE}

Dans le cas général où les paramètres $s, d$ et $a$ dépendent de $i$ et $n$, il n'est pas possible d'obtenir les probabilités stationnaires sous une forme explicite simple. Par contre, ceci peut être envisagé dans certains cas particuliers encore 
que, au niveau des applications, il n'est pas évident qu'une formule explicite soit plus utile qu'un algorithme de calcul aussi simple que celui proposé au paragraphe $D$.

Il est bien connu que, pour obtenir de telles formules explicites, la fonction génératrice peut être un intermédiaire adéquat quand les taux de services $s(n)$ ne dépendent pas de $n$. Nous allons donc nous placer dans l'un des deux cas suivants :

Premier cas : Le réseau est ouvert et le taux d'arrivée $s(i, j, n)=: s$ est fixe (indépendant de $i, j$, et $n$ ).

Deuxième cas : Le nombre total $(B-1)$ de clients est fini; pour $n \geqq B$, $s(i, j, n)=0$ et, pour $1 \leqq n \leqq B, s(i, j, n)=: s$ est fixe (indépendant de $i$, $j$ et $n)$. On a donc $p(i, j, n)=0$ pour $n>B$.

Dans les deux cas, $a(i, n)=: a(i)$ et $d(i, j, n)=: d(i, j)$ ne dépendent pas de $n$.

On pose alors :

$$
G(i, j):=\sum_{n=2}^{\infty} p(i, j, n) Z^{n-1}
$$

Rappelons que le cas $n=1$ correspond à une station vide : pour $i=j=1$, $G(i, j)$ n'est donc pas exactement la fonction génératrice.

En multipliant par $Z^{n-1}$ et en sommant, les équations (1), (2) et (3) du paragraphe $D$ impliquent :

Pour $i \neq 1$ et $k \neq m(i)$ :

$$
[d(i, k)+s] G(i, k)=s Z G(i, k)+d(i, k+1) G(i, k+1) .
$$

Pour $i \neq 1$ et $k=m(i)$ :

$$
[d(i, m(i))+s] G[i, m(i)]=s Z G[i, m(i)]+a(i) G(1,1) .
$$

Pour $i=1$ ce qui implique $k=1$ :

$$
\begin{aligned}
& {[d(1,1)+s][G(1,1)+1]=s Z[G(1,1)+1]} \\
& +a(1) Z^{-1} G(1,1)+\sum_{i=2}^{I} d(i, 1) G(i, 1) .
\end{aligned}
$$

L'équation (1)' peut s'écrire :

$$
G(i, k)=G(i, k+1) \frac{d(i, k+1)}{d(i, k)+s(1-Z)} .
$$

vol. $19, \mathrm{n}^{\circ} 3$, août 1985 
Ceci et l'équation (2)' impliquent :

$$
G(i, j)=G(1,1) \prod_{k=j}^{m(i)} \frac{d(i, k+1)}{d(i, k)+s(1-Z)},
$$

en posant $d[i, m(i)+1]:=a(i)$.

En reportant ces valeurs pour $j=1$, dans l'équation (3)', il vient :

$$
G(1,1)=[d(1,1)+s(1-Z)] /\left[\frac{a(1)}{Z}+\varphi(Z)-d(1,1)-s(1-Z)\right] .
$$

en ayant posé :

$$
\varphi(Z):=\sum_{i=2}^{I} d(i, 1) \prod_{k=1}^{m(i)} \frac{d(i, k+1)}{d(i, k)+s(1-Z)} .
$$

L'équation (7) donne alors toutes les fonctions génératrices.

\section{CONCLUSION}

Il est évident qu'on peut donner d'autres variantes de la modélisation avec " pivot ». Par exemple, on peut décider que la fin du service d'un client se situe à l'instant où l'" état fictif quitte l'état pivot " (au lieu de l'instant où l'« état fictif atteint l'état pivot »). On peut aussi décomposer l'état pivot en autant d'état fictifs qu'il n'y a de " chaînes ", etc.

Ces variantes modifient les équations mais ne modifient pas fondamentalement la méthodologie.

Par ailleurs, il est clair que cette modélisation « avec pivot » peut être utilisée pour d'autres réseaux que celui considéré ici. Il faut toutefois constater que, même avec la méthode simplificative proposée ici, le calcul des probabilités stationnaires d'un réseau de files d'attente comportant une station à loi de service quelconque devient complexe dès qu'il y a plus de deux stations dans le réseau et que les taux de service dépendent à la fois du nombre de clients et de l'« état fictif » du serveur.

\section{BIBLIOGRAPHIE}

[Coh] J. W. Cohen, The Single Server Queue, North Holland, 2nd edition, 1982.

[Cox] D. R. Cox, A Use of Complex Probabilities in the Theory of Stochastic Processes, Proc. Cambridge Philosophical Society, vol. 51, 1955, p. 313-319. 
[GeP] E. Gelenbe et G. Pujolle, Introduction aux réseaux de files d'attente, Eyrolles, Paris, 1982.

[Ke1] F. P. Kelly, Reversibility and stochastic networks, J. Wiley, 1979.

[Leb] J. Y. LE Boudec, Étude générale d'un réseau constitué de deux stations hyperexponentielles, Rapport Irisa, $\mathrm{n}^{\circ} 200$, mai 1983.

[Mar] R. MARIE, Modélisation par réseaux de files d'attente, Thèse d'État, Université de Rennes, France, novembre 1978.

[MaP-1] R. Marie et J. Pellaumail, Steady-State Probabilities for a Queue with a General Service Distribution on State-dependent Arrivals, I.E.E.E.-T.S.E., vol. SE-9, $\mathrm{n}^{\circ} 1$, janvier 1983.

[MaP-2] R. Marie et J. Pellaumail, Régime stationnaire pour une file $M / H / 1$ avec impatience, Rapport Irisa, $\mathrm{n}^{\circ} 205$, avril 1983.

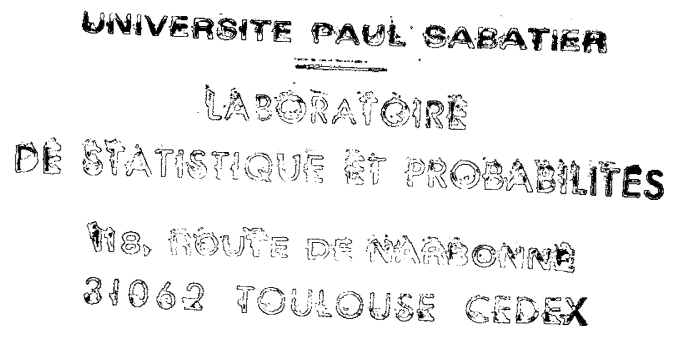

vol. $19, \mathrm{n}^{\circ} 3$, août 1985 